\title{
Lifestyle is associated with thyroid function in subclinical hypothyroidism: a cross- sectional study
}

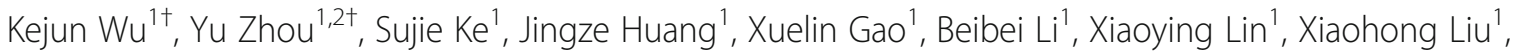

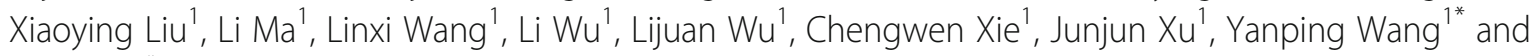
Libin Liu ${ }^{1 *}$

\begin{abstract}
Background: Few studies have focused on the association between lifestyle and subclinical hypothyroidism (SCH). The purpose of this study was to investigate the association between lifestyle and thyroid function in $\mathrm{SCH}$.

Methods: This study was a part of a community-based and cross-sectional study, the Epidemiological Survey of Thyroid Diseases in Fujian Province, China. A total of 159 participants with SCH (81 males and 78 females) and 159 euthyroid (87 males and 72 females) participants without any missing data were included in the analysis. General information and lifestyle information including sleep, exercise, diet and smoking habits of the participants was collected by questionnaire and Pittsburgh sleep quality index scale (PSQI) was collected. Thyroid stimulating hormone (TSH), free thyroxine (FT4), thyroid peroxidase antibody (TPOAb), thyroid globulin antibody (TgAb) and urine iodine concentration (UIC) were tested. Thyroid homeostasis parameter thyroid' s secretory capacity (SPINAGT), Jostel's TSH index (TSHI), thyrotroph T4 sensitivity index (TTSI) were calculated. Logistic regression and multiple linear regression were performed to assess associations.

Results: Compared with euthyroid subjects, patients with $\mathrm{SCH}$ were more likely to have poor overall sleep quality (15.1 vs.25.8\%, $P=0.018$ ) and I less likely to stay up late on weekdays (54.7 vs. $23.9 \% P<0.001)$. In SCH group, exercise was the influencing factor of TSH $(\beta=-0.224, P=0.004)$, thyroid secretory capacity $(\beta=0.244, P=0.006)$ and thyrotropin resistance $(\beta=0.206, P=0.009)$. lodine excess was the influencing factor of thyroid secretory capacity $(\beta=0.209, P=0.001)$ and pituitary thyroid stimulating function $(\beta=0.167, P=0.034)$. Smoking was the influencing factor of pituitary thyroid stimulating function $(\beta=0.161, P=0.040)$. Staying up late on weekends was the influencing factor of thyroid secretory capacity $(\beta=0.151, P=0.047)$. After adjusting for possible confounders, logistic regression showed that those with poor overall sleep quality assessed by PSQI and iodine excess had an increased risk of SCH (OR 2.159, $95 \% \mathrm{Cl} 1.186-3.928, P=0.012$ and OR 2.119, $95 \% \mathrm{Cl} 1.008-4.456, P=0.048$, respectively).
\end{abstract}

\footnotetext{
* Correspondence: yp1014wang@163.com; libinliu@fjmu.edu.cn

† Kejun Wu and Yu Zhou contributed equally to this work.

${ }^{1}$ Department of Endocrinology and Metabolism, Fujian Medical University

Union Hospital, 29 Xinquan Road, Fujian 350001 Fuzhou, China

Full list of author information is available at the end of the article
}

(c) The Author(s). 2021 Open Access This article is licensed under a Creative Commons Attribution 4.0 International License, which permits use, sharing, adaptation, distribution and reproduction in any medium or format, as long as you give appropriate credit to the original author(s) and the source, provide a link to the Creative Commons licence, and indicate if changes were made. The images or other third party material in this article are included in the article's Creative Commons licence, unless indicated otherwise in a credit line to the material. If material is not included in the article's Creative Commons licence and your intended use is not permitted by statutory regulation or exceeds the permitted use, you will need to obtain permission directly from the copyright holder. To view a copy of this licence, visit http://creativecommons.org/licenses/by/4.0/ The Creative Commons Public Domain Dedication waiver (http://creativecommons.org/publicdomain/zero/1.0/) applies to the data made available in this article, unless otherwise stated in a credit line to the data. 
Conclusions: Lifestyle including sleep, smoking, diet and exercise was closely related to thyroid function especially thyroid homeostasis in $\mathrm{SCH}$.

Keywords: Subclinical hypothyroidism, Lifestyle, Thyroid function, Cross-sectional study

\section{Background}

Subclinical hypothyroidism (SCH) refers to the abnormal thyroid function with increased serum thyrotropin (TSH) level and normal total thyroxine (TT4) and free thyroid hormone (FT4) levels. The prevalence of SCH ranges from 4.6 to $16.7 \%$ around the worldwide [1]. For lacking of obvious clinical manifestations such as fear of cold, constipation, apathy and depression, $\mathrm{SCH}$ is mainly found accidentally in routine physical examination and is easy to be ignored by patients. About $5 \%$ of $\mathrm{SCH}$ may gradually progress to clinical hypothyroidism without treatment; many studies have suggested that increased TSH is associated with cardiovascular disease risk, insulin resistance, and metabolic syndrome $[2,3]$. Therefore, $\mathrm{SCH}$ needs to be paid enough attention and corresponding measures should be taken to reduce its harm.

Lifestyle is associated with a variety of endocrine and metabolic diseases, such as obesity, dyslipidemia, metabolic syndrome and type 2 diabetes. Among thyroid diseases, researchers have pay attention to the relationship between lifestyle and $\mathrm{SCH}$. Studies have shown that changes in smoking habits could affect the occurrence of clinical hypothyroidism and $\mathrm{SCH}$ [1]; iodine deficiency and iodine excess both increase the incidence of overt hypothyroidism and $\mathrm{SCH}$ [4]; compared with sleep duration of 7-8 h, shorter and longer sleep duration was associated with an increased risk of $\mathrm{SCH}$ [5]; people who were sedentary had higher levels of TSH than those who exercised regularly [6], and $\mathrm{SCH}$ was associated with decreased exercise tolerance [7].However,. there are few studies on how lifestyle affects thyroid function in $\mathrm{SCH}$. Thyroid function is regulated by hypothalamus and pituitary. The changes of thyroid hormone are regulated by the negative feedback of hypothalamus pituitary thyroid (HPT) axis. We speculate that for $\mathrm{SCH}$, the dysfunction of HPT axis may occur before TSH increasing. Basic thyroid functional parameters like TSH and T4 may not reflect the dynamic change of HPT axis accurately. Therefore, novel parameters obtained by mathematical modeling were created as supplementary indicators [810]. Thyroid homeostasis parameters reflect the functional status of HPT axis, which can be used as a new perspective to evaluate the impact of lifestyle on thyroid function in patients with $\mathrm{SCH}$.

Therefore, the purpose of this study was to investigate the association between lifestyle and thyroid function in $\mathrm{SCH}$ by analyzing the correlation between $\mathrm{SCH}$ and lifestyle including sleep quality and habit, exercise, diet and smoking, and the association between lifestyle and thyroid homeostasis in patients with $\mathrm{SCH}$.

\section{Methods \\ Study population}

This cross-sectional study was conducted in 2016 in the Fujian Province of China. All the participants enrolled the national epidemiological survey (Tide) aimed to determine the prevalence of thyroid diseases and diabetes and the iodine nutrition status[11]. The subjects were enrolled according to the following criteria: (1) adult residents aged over 18 years; (2) Han race; (3) and living in Fujian for at least 5 years. The exclusion criteria were as follows: (1) receiving iodine contrast agent examination or taking iodine-containing drugs in recent three months; (2) pregnant and breastfeeding women. A total of 2,651 individuals were randomly selected from the general population in urban $(n=1394)$ and rural $(n=1257)$ areas. For further analysis of population with $\mathrm{SCH}$, subjects with elevated serum TSH levels and normal FT4 levels were considered as subclinical hypothyroidism [12]. The following were exclusion criteria: (1) patients with previously diagnosed thyroid diseases, including hyperthyroidism, hypothyroidism, thyroid tumors, thyroid nodules, etc., regardless of whether they have been treated; (2) patients with normal thyroid function, but with morbid syndrome acute and chronic renal insufficiency, adrenocortical hypofunction and other diseases that may cause abnormal increase of TSH. The study was carried in accordance to Declaration of Helsinki. The protocol was approved by the Ethics Committee of Fujian Medical University Union Hospital (Grant no.2015KY032). Subjects with high TSH levels (> $4.2 \mathrm{mIU} / \mathrm{L})$ and normal free T4 (FT4) levels (12.0-22.0 $\mathrm{pmol} / \mathrm{L})$ were diagnosed with $\mathrm{SCH}(n=214)$. Subjects with a history of thyroid disease treatment, such as surgery, radiation, and thyroid hormone or antithyroid medication $(\mathrm{n}=21)$ and with missing data $(\mathrm{n}=34)$ were further excluded. There were 159 participants with, including 81 males and 78 females (mean age $=$ $42.4 \pm 15.8$ years) in the final analysis. Age and gender matched subjects were selected from normal TSH population participating in this epidemiological investigation as the control group by propensity score matching. The control group consisted in 159 euthyroid (EUTH) subjects, including 87 males and 72 females (mean age $=40.6 \pm 15.0$ years) without previous history of thyroid disease and treatment and missing data. 


\section{Demographic and anthropometric variables}

The general data such as name, sex, age, occupation, education level and past medical history were collected by questionnaire. The data of height, weight, waist circumference (WC), systolic blood pressure (SBP), diastolic blood pressure (DBP) were measured. Height and weight were measured in light clothing without shoes. Waist circumference (WC) was measured using a tape measure placed halfway between the lower border of the ribs and the iliac crest in a horizontal plane. Height, weight and $\mathrm{WC}$ were measured twice and the averages were taken. Height, $\mathrm{WC}$ and weight were measured to the nearest $0.1 \mathrm{~cm}$ and $0.1 \mathrm{~kg}$, respectively. Body mass index (BMI) was calculated by dividing the participant's weight $(\mathrm{kg})$ by the square of height $\left(\mathrm{m}^{2}\right)$.

\section{Laboratory measurements}

After fasting for $8 \mathrm{~h}$ at night, the venous blood samples were collected between 08:00 am to 10:00 am. Thyroid hormones and thyroid autoantibodies, including thyroid stimulating hormone (TSH), free thyroxine (fT4), and free triiodothyronine (fT3) thyroid peroxidase antibody (TPOAb), thyroid globulin antibody (TgAb) were quantified using the immunochemiluminometric assay (ICMA) method, using the standard kits from Roche Diagnostics GmbH (Germany). To screen the prevalence of thyroid disorders in the population, fT4 and fT3 were measured only among those with abnormal levels of TSH. Fasting blood glucose (FBG), serum total cholesterol (TC), triglyceride (TG), high-density lipoprotein cholesterol (HDL-C), low-density lipoprotein cholesterol (LDL-C), and uric acid (UA) were measured by an automated procedure (BS180; Mindray, Ltd., Shenzhen, China). Hemoglobin ( $\mathrm{Hb}$ ) A1c was measured by HPLC using an automated analyzer (V ARIANT ${ }^{\mathrm{TM}}$ II TURBO, BioRad, Berkeley, USA). Midstream urine samples were collected in the morning, and urinary iodine concentration (UIC) was quantified using the ammonium persulfate method. According to the standard put forward by WHO/UNICEF/ICCIDD in 2007 [13], UIC > $300 \mu \mathrm{g} / \mathrm{L}$ is regarded as iodine excess.

\section{Thyroid homeostasis assessment}

Thyroid homeostasis parameter thyroid' s secretory capacity (SPINA-GT), Jostel's TSH index (TSHI), thyrotroph T4 sensitivity index (TTSI, also referred to thyrotroph thyroid hormone resistance index or thyrotroph thyroxine resistance index) were calculated.

SPINA-GT provides an estimate of the maximum thyroid secretory rate under stimulation conditions. The maximum stimulating effect of TSH on the thyroid and the dissociation, protein binding, distribution and elimination of FT4 are involved in SPINA-GT. It was sensitive to thyroid diseases of primary origin and specific for secondary dysfunction of thyroid [9]. Previous studies have observed that SPINA-GT correlates with thyroid volume measured by ultrasound, and increases in hyperthyroidism and decreases in hypothyroidism [14]. It was calculated as

$$
\widehat{G}=\frac{\beta_{T}\left(D_{T}+[T S H]\right)\left(1+K_{41}[T B G]+K_{42}[T B P A]\right)\left[F T_{4}\right]}{\alpha_{T}[T S H]}
$$

In this formular, TBG referred to standard concentration of thyroxine-binding globulin, and TBPA referred to standard transthyretin concentration. Constants in these formulas were as follows: $\beta_{\mathrm{T}}=1.1 \times 10^{-6} / \mathrm{s}, \mathrm{D}_{\mathrm{T}}=2.75$ $\mathrm{mU} / \mathrm{L}, \mathrm{K}_{41}=2.0 \times 10^{10} \mathrm{~L} / \mathrm{mol}, \mathrm{TBG}=300 \mathrm{nmol} / \mathrm{L}, \mathrm{K}_{42}=$ $2.0 \times 10^{8} \mathrm{~L} / \mathrm{mol}, \mathrm{TBPA}=4.5 \mu \mathrm{mol} / \mathrm{L}$ and $\alpha_{\mathrm{T}}=0.1 / \mathrm{L}$.

The function of the pituitary was evaluated by TSHI. It estimated the maximum pituitary TSH reserve by correcting the negative feedback suppression of TSH by measured FT4 concentration and extrapolating the standardized uninhibited TSH assuming a FT4 value of 0 , providing an accurate estimate of the severity of pituitary dysfunction. As a supplementary indicator of pituitary function, it provided a sufficiently accurate and sensitive assessment of the severity of hypopituitary function by basic thyroid function test in the absence of dynamic pituitary function test[15]. Its reduction was thought to be associated with gonadotropin insufficiency, lower peak concentrations of growth hormone and cortisol in pituitary stimulation tests, and it was used in the assessment of non-thyroid disease syndroms (NTIS) [9]. It was calculated as

$$
T S H I=\ln ([\mathrm{TSH}])+0.1345 \times\left[\mathrm{FT}_{4}\right]
$$

An additional index used for assessing thyrotropic function and TSH feedback inhibition was TTSI. It was calculated by the equilibrium concentrations of TSH and free $\mathrm{T} 4$ and the upper limit of the reference interval of FT4 $\left(l_{u}\right)$ [9]. It represented the feedback of the HPT axis, and its elevation was associated with thyroid hormone resistance and pituitary dysregulation. It has been used to evaluate the dysregulation of the HPT axis due to genetic mutations and genetic factors $[16,17]$. It was calculated as

$$
T T S I=\frac{100[T S H]\left[F T_{4}\right]}{l_{u}}
$$

\section{Assessment of lifestyle}

Lifestyle data including sleep, diet, smoking and exercise were collected by questionnaire. The Pittsburg sleep quality index scale (PSQI) was used to assess the overall sleep quality of subjects. PSQI is a sleep quality scale compiled by Buysse et al. in 1989 [18] which has a high correlation with the results of polysomnography and is 
simple and feasible with high reliability and validity. PSQI includes seven dimensions: subjective sleep quality, sleep latency, sleep duration, habitual sleep efficiency, sleep disturbances, use of sleeping medications, and daytime dysfunction. The total score of PSQI $\geq 7$ in this study was regarded as poor overall sleep quality. Living habits were collected by questionnaire including: (1) sleep habits: lunch break (more than $30 \mathrm{~min}$ or not), bedtime at night on weekdays and weekends (later than 10:00 pm or not), Sleep duration on weekdays and weekends (less than $5 \mathrm{~h}, 5-6 \mathrm{~h}, 7-8 \mathrm{~h}, 9-10 \mathrm{~h}$ and more than $10 \mathrm{~h}$ ); (2) exercise habits: exercise for more than 3 days a week, lasting more than 30 min once (no, low intensity and medium or high intensity); (3) eating habits: daily salt intake (less than $5 \mathrm{~g} 5 \sim 10 \mathrm{~g}$, more than $10 \mathrm{~g}$ ), type of salt intake (iodized salt or not), iodine-rich foods intake like kelp and laver (none, monthly and daily). (4) and smoking habit (yes or no).

\section{Statistical analysis}

All the statistical analyses were performed by the SPSS version 26.0 (IBM SPSS Inc.). The continuous variables were reported as mean values and standard deviations or as median values and corresponding 25th and 75th percentiles. The percentages were reported for the categorical variables. Student's T test or Mann-Whitney U test was used to analyze the differences in continuous variables between $\mathrm{SCH}$ group and NC group. Chi-squared test or Fisher's exact test were performed to assess differences in categorical variables between the two groups. Because TTSI levels were distributed in a skewed manner, they were reciprocally transformed before statistical analysis. Correlation between thyroid homeostasis variables and thyroid homeostasis parameters were calculated using the Pearson or Spearman correlation coefficient. Logistic regression was used to analyze the risk factors of $\mathrm{SCH}$. Age, gender, BMI, SBP, TgAb, TC, UA and HbA1c were included in logistic regression as confounding factors to evaluate whether a risk factor was independently associated with $\mathrm{SCH}$. Multiple linear regression was used to further analyze the influence of lifestyle on thyroid homeostasis parameters in $\mathrm{SCH}$ group. Statistically significant factors were selected from correlation analysis as independent variables, and the independent variables were screened by forward method to establish the model. The lifestyle variables with statistically significant $(P<0.05)$ such as exercise, stay up late on weekends, iodine excess and smoking were included in the model, that is, the influencing factors of thyroid homeostasis parameters. $P<0.05$ was considered to be statistically significant.

\section{Results}

\section{General characteristics}

There were 159 participants in EUTH group and $\mathrm{SCH}$ group respectively. General characteristics of the population are as shown in Table 1. There were no significant difference in age and gender between EUTH subjects (mean age $40.6 \pm 15.0$ years, females $45.3 \%$ ) and patients with SCH (mean age $42.4 \pm 15.8$ years, females $49.1 \%$ ).Compared with euthyroid subjects, patients with $\mathrm{SCH}$ were more likely to have poor overall sleep quality (15.1 vs. $25.8 \% P=0.018)$ and less likely to stay up late on weekdays ( 54.7 vs. $23.9 \% P<0.001)$. There was significant different in sleep duration on weekends categorized into less than $5 \mathrm{~h}, 5 \sim 6 \mathrm{~h}, 7 \sim 8 \mathrm{~h}, 9 \sim 10 \mathrm{~h}$ and more than $10 \mathrm{~h}$ between EUTH subjects and patients with $\mathrm{SCH}(1.3,15.1,59.1,24.5,0 \%$ vs. $1.9,20.8,61.6$, $11.9,3.8 \%, P<0.001)$. TSH, positive rate of TPOAb and $\mathrm{TgAb}$ were significantly increased in patients with $\mathrm{SCH}$ compared with EUTH subjects $(5.01 \mathrm{mIU} / \mathrm{L}$ vs.1.92 $\mathrm{mIU} / \mathrm{L} P<0.001,19.5 \%$ vs. $8.8 \% P=0.006$ and $26.4 \%$ vs. $6.3 \% P<0.001$ respectively). There was no significant difference in lunch break, stay up late on weekends, sleep duration on weekdays, exercise, dietary habits related to iodine intake, UIC and smoking between $\mathrm{SCH}$ group and NC group $(P>0.05)$. There was also a trend towards higher BMI $\left(23.9 \pm 3.2 \mathrm{~kg} / \mathrm{m}^{2}\right.$ vs. $22.6 \pm 3.1 \mathrm{~kg} /$ $\left.\mathrm{m}^{2}, P<0.001\right)$, WC $(81.7 \pm 9.5 \mathrm{~cm}$ vs. $77.8 \pm 10.6 \mathrm{~cm}$, $P=0.001), \quad$ SBP $(127.0 \pm 19.0 \mathrm{mmHg}$ vs. $122.3 \pm 16.2$ $\mathrm{mmHg}, P=0.019)$ and DBP $(80.0 \pm 11.6 \mathrm{mmHg}$ vs. $76.4 \pm 10.8 \mathrm{mmHg}, P=0.013$ ), in patients with $\mathrm{SCH}$ compared with EUTH subjects. The subjects in $\mathrm{SCH}$ group had higher FBG, HbA1c, TG, TC, LDL-C, UA and lower HDL-C than that in EUTH group although there was no significant difference between the two groups. Comparison of sleep quality between EUTH group and $\mathrm{SCH}$ group are as shown in Fig. 1. The total scores of PSQI of patients with $\mathrm{SCH}$ were significantly higher than those of EUTH subjects $(4.50 \pm 2.83$ vs. $3.88 \pm 2.29, P=0.034)$. Among the seven dimensions of PSQI, the scores of subjective sleep quality of patients with $\mathrm{SCH}$ were significantly higher than those of EUTH subjects $(1.10 \pm 0.61$ vs. $0.94 \pm 0.57, P=0.018$ ), and the scores of sleep latency of patients with $\mathrm{SCH}$ were significantly higher than those of EUTH subjects $(0.70 \pm 0.88$ vs. $0.94 \pm 0.57, P=$ $0.003)$. There was no significant difference in sleep duration, habitual sleep efficiency, sleep disturbances, sleep medication use, and daytime dysfunction between the two groups.

Association between lifestyle and thyroid function in $\mathrm{SCH}$ Spearman correlation analysis showed that in $\mathrm{SCH}$ group, TSH was negatively correlated with exercise $(r=$ -0.309, $P<0.001)$, SPINA-GT was positively correlated with stay up late on weekends $(r=0.163, P=0.040)$, 
Table 1 Characteristics of subjects with and without subclinical hypothyroidism

\begin{tabular}{|c|c|c|c|}
\hline Characteristics & EUTH $(n=159)$ & $\mathrm{SCH}(n=159)$ & ${ }^{*} P$ value \\
\hline Female, \% & 45.3 & 49.1 & 0.500 \\
\hline Age, years & $40.6 \pm 15.0$ & $42.4 \pm 15.8$ & 0.297 \\
\hline \multicolumn{4}{|l|}{ Anthropometric variables } \\
\hline $\mathrm{BMI}, \mathrm{kg} / \mathrm{m}^{2}$ & $22.6 \pm 3.1$ & $23.9 \pm 3.2$ & $<0.001$ \\
\hline$W C, \mathrm{~cm}$ & $77.8 \pm 10.6$ & $81.7 \pm 9.5$ & 0.001 \\
\hline $\mathrm{SBP}, \mathrm{mmHg}$ & $122.3 \pm 16.2$ & $127.0 \pm 19.0$ & 0.019 \\
\hline $\mathrm{DBP}, \mathrm{mmHg}$ & $76.4 \pm 10.8$ & $80.0 \pm 11.6$ & 0.013 \\
\hline \multicolumn{4}{|l|}{ Laboratory measurements } \\
\hline $\mathrm{TSH}, \mathrm{mlU} / \mathrm{L}$ & $1.92(1.42,2.51)$ & $5.01(4.60,6.13)$ & $<0.001$ \\
\hline TPOAb,$+ \%$ & 8.8 & 19.5 & 0.006 \\
\hline $\operatorname{TgAb}+, \%$ & 6.3 & 26.4 & $<0.001$ \\
\hline $\mathrm{UIC}, \mu \mathrm{g} / \mathrm{L}$ & $140.70(89.28,222.40)$ & $152.20(99.73,227.90)$ & 0.275 \\
\hline $\mathrm{FBG}, \mathrm{mmol} / \mathrm{L}$ & $5.04(4.69,5.66)$ & $5.17(4.80,5.50)$ & 0.262 \\
\hline $\mathrm{HbA} 1 \mathrm{c}, \%$ & $5.8 \pm 0.9$ & $5.9 \pm 1.0$ & 0.472 \\
\hline $\mathrm{TG}, \mathrm{mmol} / \mathrm{L}$ & $0.98(0.69,1.37)$ & $1.08(0.81,1.59)$ & 0.102 \\
\hline $\mathrm{TC}, \mathrm{mmol} / \mathrm{L}$ & $4.88 \pm 1.09$ & $5.09 \pm 1.19$ & 0.087 \\
\hline $\mathrm{HDL}-\mathrm{C}, \mathrm{mmol} / \mathrm{L}$ & $1.37 \pm 0.40$ & $1.35 \pm 0.38$ & 0.151 \\
\hline $\mathrm{LDL}-\mathrm{C}, \mathrm{mmol} / \mathrm{L}$ & $2.44 \pm 0.70$ & $2.57 \pm 0.79$ & 0.712 \\
\hline UA, $\mathrm{mmol} / \mathrm{L}$ & $340.35 \pm 95.15$ & $346.59 \pm 92.61$ & 0.554 \\
\hline \multicolumn{4}{|l|}{ Lifestyle information } \\
\hline $\mathrm{PSQI} \geq 7, \%$ & 15.1 & 25.8 & 0.018 \\
\hline More than 30 min lunch break, \% & 44.7 & 52.8 & 0.145 \\
\hline Stay up late on weekdays, \% & 54.7 & 23.9 & $<0.001$ \\
\hline Stay up late on weekends, \% & 67.3 & 62.9 & 0.410 \\
\hline Sleep duration on weekdays $(<5 \mathrm{~h} / 5 \sim 6 \mathrm{~h} / 7 \sim 8 \mathrm{~h} / 9 \sim 10 \mathrm{~h} />10 \mathrm{~h}), \%$ & 1.9/27.7/57.9/12.6/0 & $4.4 / 32.7 / 52.2 / 8.2 / 2.5$ & 0.087 \\
\hline Sleep duration on weekends ( $<5 \mathrm{~h} / 5 \sim 6 \mathrm{~h} / 7 \sim 8 \mathrm{~h} / 9 \sim 10 \mathrm{~h} />10 \mathrm{~h}$ ), \% & $1.3 / 15.1 / 59.1 / 24.5 / 0$ & 1.9/20.8/61.6/11.9/3.8 & 0.003 \\
\hline Exercise and intensity (no/low/medium or high), \% & 38.4/44.0/17.6 & $42.1 / 35.2 / 14.5$ & 0.242 \\
\hline Daily salt intake $(<5 \mathrm{~g} / 5 \sim 10 \mathrm{~g} />10 \mathrm{~g}), \%$ & $14.5 / 73 / 12.6$ & $14.5 / 73 / 12.6$ & 0.759 \\
\hline lodized salt intake, \% & 95.0 & 89.3 & 0.061 \\
\hline lodine-rich foods intake (none/monthly/daily), \% & 11.9/70.4/17.6 & 13.2/71.1/15.7 & 0.872 \\
\hline Smoking, \% & 18.9 & 16.4 & 0.556 \\
\hline
\end{tabular}

* $P$ value $<0.05$ was considered significant

Data are shown as mean (SD), median (interquartile range), or percentage

PSQI Pittsburg sleep quality index scale; UIC urinary iodine concentration; TSH thyroid stimulating hormone; TPOAb thyroid peroxidase antibody; TgAb thyroid globulin antibody; $B M I$ body mass index; WC waist circumference; SBP systolic blood pressure; DBP diastolic blood pressure; FBG fasting blood glucose; HbA1c glycosylation hemoglobin; TG triglyceride; TC total cholesterol; HDL-C high density lipoprotein cholesterol; $L D L-C$ low density lipoprotein cholesterol; UA uric acid

exercise $(r=0.197, P=0.013)$, smoking $(r=0.196, P=$ $0.013)$ and iodine excess $(r=0.216, P=0.006)$, and negatively correlated with eating iodine-rich foods intake $(r=-$ 0.164, $P=0.039$ ); TSHI was positively correlated with smoking $(r=0.166, P=0.036)$ and iodine excess $(r=$ $0.191, P=0.016)$; and TTSI $^{-1}$ was positively correlated with exercise $(r=0.223, P=0.005) P<($ Table 2$)$. Multiple linear regression analysis showed that in the $\mathrm{SCH}$ group, the independent variables iodine excess $(\beta=0.209, P=$
$0.001)$, exercise $(\beta=0.244, P=0.006)$, and stay up late on weekends $(\beta=0.151, P=0.047)$ were included in the model of SPINA-GT. The independent variables smoking $(\beta=0.161, P=0.040)$ and iodine excess $(\beta=0.167$, $P=0.034)$ were included in the model of TSHI. The regression type of $\mathrm{TTSI}^{-1}$ was statistically significant, and The independent variable exercise $(\beta=-0.224, P=0.004$ and $\beta=0.206, P=0.009$, respectively) was included in the model of TSH and $\mathrm{TTSI}^{-1}$. All the models above 


\section{EUTH}

- $\mathrm{SCH}$

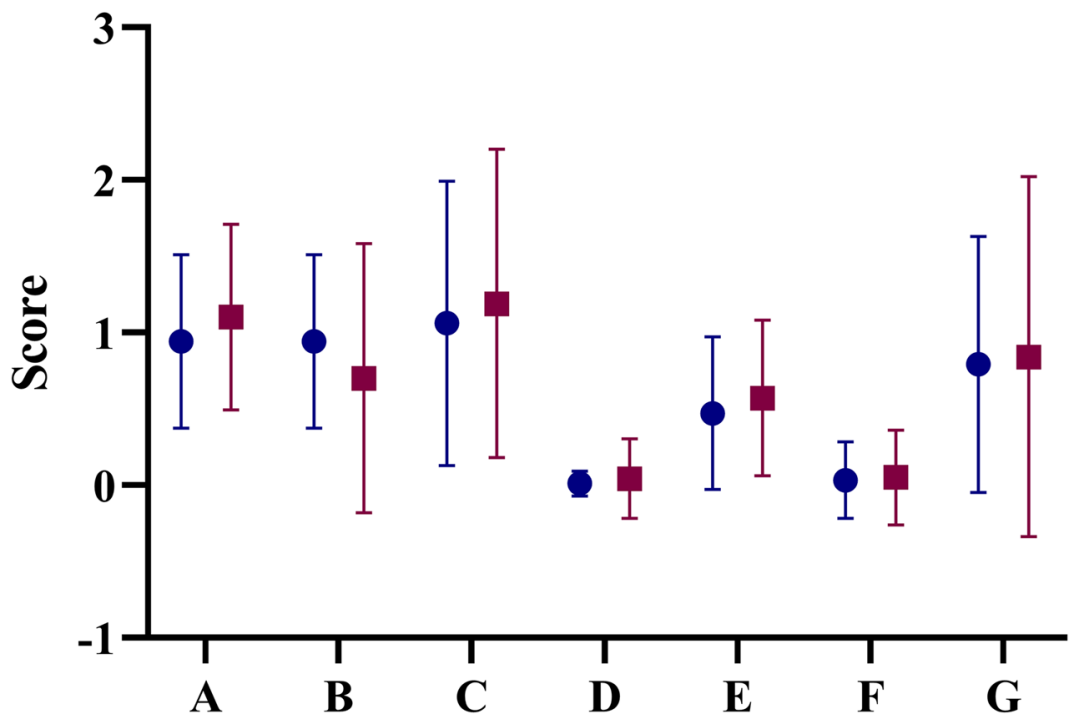

Fig. 1 Score of seven dimensions of the Pittsburg sleep quality index scale (PSQI). A, subjective sleep quality; B, sleep latency; C, sleep duration; D, habitual sleep efficiency; E, sleep disturbances; F, use of sleeping medications; G, daytime dysfunction

were statistically significant (all $P<0.05$ ).It was suggested that the effects of the above variables on TSH and thyroid homeostasis parameters were statistically significant $P<$ (Table 3).

\section{Lifestyle factors associated with SCH}

To identify lifestyle factors associated with $\mathrm{SCH}$, we analyzed age, sex, physical indicators, thyroid autoantibody and lifestyle factors in a logistic regression model. For the collinearity existed between BMI and WC, SBP and $\mathrm{DBP}$, and TPOAb and TgAb, BMI, SBP and TgAb were selected to represent obesity index, blood pressure level and thyroid autoimmunity respectively. In model $1 \mathrm{ad}-$ justed age, sex, BMI, SBP and TgAb, logistic regression showed that those with poor overall sleep quality assessed by PSQI had an increased risk of SCH (OR 2.138, $95 \%$ CI 1.176-3.886, $P=0.013$ ), and those who slept late on weekdays had a lower risk of SCH (OR

Table 2 Correlation between lifestyle and thyroid homeostasis in subclinical hypothyroidism

\begin{tabular}{|c|c|c|c|c|c|c|c|c|}
\hline \multirow[t]{2}{*}{ Parameters } & \multicolumn{2}{|l|}{ TSH } & \multicolumn{2}{|c|}{ SPINA-GT } & \multicolumn{2}{|l|}{ TSHI } & \multicolumn{2}{|c|}{$\mathrm{TTSI}^{-1}$} \\
\hline & $r$ & $P$ value & $r$ & $P$ value & $r$ & $P$ value & $r$ & $P$ value \\
\hline PSQI score & -0.039 & 0.626 & 0.050 & 0.533 & -0.017 & 0.830 & 0.043 & 0.588 \\
\hline More than 30 min lunch break & -0.062 & 0.440 & 0.094 & 0.238 & 0.020 & 0.798 & 0.017 & 0.832 \\
\hline Stay up late on weekdays & -0.099 & 0.213 & 0.124 & 0.120 & 0.042 & 0.060 & 0.006 & 0.939 \\
\hline Stay up late on weekends & -0.148 & 0.063 & 0.163 & 0.040 & 0.052 & 0.513 & 0.014 & 0.865 \\
\hline Sleep duration on weekdays & 0.133 & 0.095 & -0.071 & 0.373 & 0.124 & 0.120 & -0.150 & 0.058 \\
\hline Sleep duration on weekends & 0.097 & 0.221 & -0.006 & 0.941 & 0.094 & 0.240 & -0.084 & 0.293 \\
\hline Exercise & -0.309 & $<0.001$ & 0.197 & 0.013 & -0.141 & 0.077 & 0.223 & 0.005 \\
\hline Daily salt intake & 0.051 & 0.525 & -0.125 & 0.118 & -0.070 & 0.382 & 0.033 & 0.683 \\
\hline lodized salt intake & -0.063 & 0.434 & -0.067 & 0.402 & -0.144 & 0.071 & 0.125 & 0.113 \\
\hline lodine-rich foods intake & 0.149 & 0.061 & -0.164 & 0.039 & -0.028 & 0.725 & -0.025 & 0.753 \\
\hline lodine excess & -0.020 & 0.806 & 0.216 & 0.006 & 0.191 & 0.016 & -0.125 & 0.117 \\
\hline Smoking & -0.039 & 0.625 & 0.196 & 0.013 & 0.166 & 0.036 & -0.104 & 0.193 \\
\hline
\end{tabular}

${ }^{*} P$ value $<0.05$ was considered significant PSQI Pittsburg sleep quality index scale 
Table 3 Multiple linear regression of lifestyle and thyroid homeostasis in subclinical hypothyroidism

\begin{tabular}{lllc}
\hline Dependent variable & Influence factors & $\boldsymbol{\beta}$ & $\boldsymbol{P}$ value \\
\hline TSH & Exercise & -0.224 & $\mathbf{0 . 0 0 4}$ \\
SPINA-GT & lodine excess & 0.209 & $\mathbf{0 . 0 0 1}$ \\
& Exercise & 0.244 & $\mathbf{0 . 0 0 6}$ \\
& Stay up late on weekends & 0.151 & $\mathbf{0 . 0 4 7}$ \\
TSHI & Smoking & 0.161 & $\mathbf{0 . 0 4 0}$ \\
& lodine excess & 0.167 & $\mathbf{0 . 0 3 4}$ \\
TTSI $^{-1}$ & Exercise & 0.206 & $\mathbf{0 . 0 0 9}$
\end{tabular}

${ }^{*} P$ value $<0.05$ was considered significant

SPINA-GT secretory capacity of the thyroid gland; TSHI Jostel's TSH index; $\pi S I$ thyrotroph thyroid hormone resistance index

$0.284,95 \%$ CI $0.170-0.473, P<0.001)$, and those who with iodine excess had a higher risk of SCH (OR 2.117, $95 \%$ CI 1.008-4.444, $P=0.047$ ). In order to further adjust the effect of metabolic factors, TC, UA and HbA1c were added in model 2, which represented blood lipid, blood uric acid and blood glucose respectively. Logistic regression showed the associations above between lifestyle factors with $\mathrm{SCH}$ remained the same (poor overall sleep quality OR 2.159, $95 \%$ CI 1.186-3.928, $P=0.012$, stay up late on weekdays OR $0.283,95 \%$ CI $0.169-0.472$, $P<0.001$ and iodine excess OR 2.119, $95 \%$ CI 1.0084.456, $P=0.048$, respectively). Lunch break, sleep duration, exercise and smoking etc. were not correlated with $\mathrm{SCH}$ under either model 1 or model 2 (Fig. 2).

\section{Discussion}

In this study, a cross-sectional study based on community population was conducted to analyze the association between lifestyle and thyroid function of $\mathrm{SCH}$ patients from the point of view of HPT axis function and thyroid homeostasis. It was suggested by the results that lifestyle including sleep, diet, exercise and smoking was closely related to the thyroid function of $\mathrm{SCH}$.

The present study suggests that compared with the euthyroid subjects, $\mathrm{SCH}$ patients had poorer overall and subjective sleep quality, shorter sleep latency and lower proportion of late sleep on weekdays. The sleep duration of $\mathrm{SCH}$ patients was different from that of EUTH group. However, there was no difference in exercise, diet and smoking habits between $\mathrm{SCH}$ group and EUTH group in this study. The relationship between lifestyle and $\mathrm{SCH}$ has been concerned by some researchers.

Previous studies have shown that lack of sleep can affect the function of the HPT axis [19]. TSH is the main hormone which affected by circadian rhythm in the HPT axis. TSH is secreted instantly during 20:00 to 03: 00 , and reaches a peak at about 02:00 to 03:00, and then decreases gradually [20]. The relationship between SCH and sleep quality can be explained by the inhibitory effect of sleep on TSH secretion. Lack of sleep is associated with a nearly two-fold increase in the level of TSH at night, suggesting that adequate and high-quality sleep during night can inhibit TSH levels, while compensatory sleep during daytime did not suppress TSH levels below the daytime levels. The mechanism may be that the HPT axis under the control of the circadian clock via the suprachiasmatic nucleus pacemaker, and compensatory sleep after sleep deprivation violates the original circadian rhythm, resulting in the secretion of TSH cannot follow the circadian cycle fluctuations[21]. However, prolonged sleep can also affect the TSH secretion, which may be related to circadian rhythm disorders. A crosssectional study suggests that shorter and longer sleep periods are associated with subclinical thyroid dysfunction compared with the optimal sleep duration 7-8 $\mathrm{h}$ per night [5]. The relationship between HPT axis function and sleep may not be one-way, and some studies have shown that the dysfunction of HPT axis can lead to a decline in sleep quality [22]. The hypothalamic suprachiasmatic nucleus is the control center of circadian rhythm [21]. It is reported that there are two forms of $\mathrm{TSH}$, free $\mathrm{TSH}$ and macro $\mathrm{TSH}$, in human body. Free TSH is synthesized in thyrotrophs of the pars distalis (PD) of the pituitary gland and stimulates synthesis and release of thyroxine. Macro TSH is secreted or complexformed at the pars tuberalis (PT) adjacent to the pituitary and is controlled by suprachiasmatic nucleus which regulates circadian rhythms [23]. The increase of serum macro TSH is related to poor sleep quality [24]. In order to evaluate the relationship between the function of HPT axis and sleep quality, thyroid homeostasis parameters such as SPINA-GT, reflecting the maximum secretory capacity of the thyroid gland [9], and TSHI and TTSI, reflecting the thyroid secretory function of the pituitary gland [10] were introduced in this study. Univariate analysis and multiple linear regression suggested that staying up late on weekends was a factor affecting thyroid secretory function in patients with $\mathrm{SCH}$. However, the $P$ values $(0.040$ and 0.047 , respectively) were close to the critical value of significance test, suggesting that further study is needed to explore the association between staying up late on weekends and thyroid secretion function.

To the best of our knowledge, there is no consensus on the correlation between thyroid function and exercise so far. Studies have shown that exercise training can improve the quality of life, sit and reach test results and carotid intima-media thickness and reduce body fat ratio in women with $\mathrm{SCH}$, but has no significant effect on thyroid-related hormones $[25,26]$. In this study, although there was no significant difference, the proportion of people with regular physical activity in control group was higher than that of $\mathrm{SCH}$ group. Patients with 


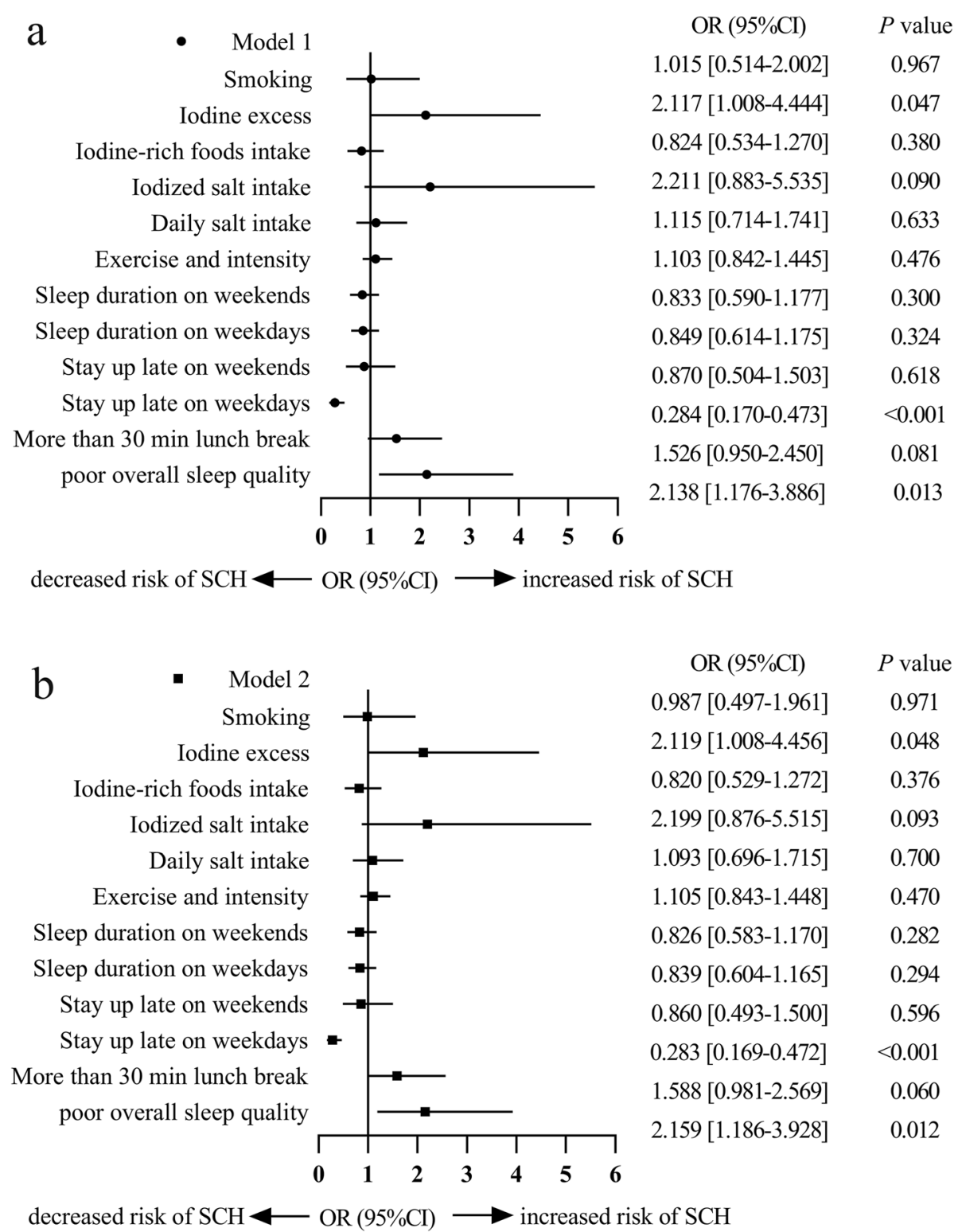

Fig. 2 The risk factor and protection factor of SCH adjusted by possible confounding factors. Model 1 adjusted age, sex, BMI, SBP, TgAb. Model 2 adjusted age, sex, BMI, SBP, TgAb. TC, UA, HbA1c. The risk of SCH in subjects with poor overall sleep quality was higher than that with good overall sleep quality. The risk of SCH in subjects with iodine excess was higher than that without iodine excess. And the risk of SCH in those who stayed up late on weekdays was lower than who slept early on weekdays $(P<0.05)$

hypothyroidism are known to have reduced excitability and inactivity due to thyroid hormone deficiency, but whether inactivity occurs in patients with $\mathrm{SCH}$ without an overt decrease in thyroid hormone has not been discussed in detail. The present study suggested that in $\mathrm{SCH}$ group, exercise intensity was negatively correlated with TSH and positively correlated with SPINA-GT and $\mathrm{TTSI}^{-1}$. Multiple linear regression showed that exercise was included in the regression model of TSH, SPINAGT and $\mathrm{TTSI}^{-1}$ respectively, suggesting that regular, moderate and high intensity exercise is related to lower
TSH, better thyroid secretion and lower thyrotropin resistance. Although the present study does not yet clarify whether patients with $\mathrm{SCH}$ show a state of inactivity before the thyroid hormone is significantly decreased, it shows the correlation between $\mathrm{SCH}$ and inactivity, as well as the correlation between moderate and high intensity exercise and better thyroid function in patients with $\mathrm{SCH}$.

Studies have shown that iodine deficiency [27] and iodine excess [28] are risk factors for hypothyroidism and $\mathrm{SCH}$. This study suggested that in $\mathrm{SCH}$ group, 
SPINA-GT and TSHI were positively correlated with iodine excess. And in multiple linear regression, iodine excess was included in the regression model of SPINAGT and TSHI respectively, suggesting that iodine excess can enhance thyroid secretion and the ability of the pituitary gland to promote thyroid secretion. Studies on rats have shown that the iodization of thyroid hormone in thyroid was inhibited when iodine was excessive, and the synthesis of thyroxine was decreased, which contributed to the increased secretion of TSH by pituitary and release of thyroid hormones that have been synthesized in the thyroid gland. As a result, the serum thyroxine level was normal while the serum TSH level was increased [29], showing the performance of $\mathrm{SCH}$. In physiological conditions, pituitary secretion of TSH promotes thyroid secretion of thyroxine, and when serum thyroxine increases, it produces negative feedback regulation on pituitary to reduce TSH secretion. It is precisely because of this negative feedback regulation that TSH and serum thyroxine levels can not accurately reflect the ability of pituitary to promote thyroid secretion and thyroid secretion[30]. It is necessary to be evaluated by thyroid homeostasis parameters. In some individuals, iodine excess can induce hypothyroidism, although thyroid function may recover after iodine withdrawal[31]. In the $\mathrm{SCH}$ group, we observed that there was a negative correlation between SPINA-GT and iodine-rich foods intake, but there was no correlation between iodine excess and iodine-rich foods intake, the possible reason was that iodine nutrition status is related to dietary iodine intake, while the frequency and amount of iodine-rich foods intake, the storage mode of iodine-rich food and the cooking method of iodine-rich foods can affect the intake of iodine [32]. Therefore, the relationship between SPINA-GT and iodine nutrition status should be evaluated by UIC rather than the frequency of iodine-rich foods intake.

Studies have shown that among women with $\mathrm{SCH}$, the average TSH level of smokers is higher than that of nonsmokers, and the clinical performance score of smokers shows that their hypothyroidism is more severe [33]. Some studies also suggested that the prevalence of elevated serum TSH was significantly decreased in smokers [34], and the risk of autoimmune-related hypothyroidism increased significantly after quitting smoking[35], suggesting that smoking may be a protective factor for hypothyroidism. This study showed that SPINA-GT and TSHI were positively correlated with smoking in $\mathrm{SCH}$ group, suggesting that smoking was related to better thyroid secretion and pituitary thyroid-promoting function. Multiple linear regression suggested that smoking was included in the regression model of TSHI, suggesting that smoking is one of the influencing factors of pituitary thyroid-promoting function.
The main cause of $\mathrm{SCH}$ is thyroid autoimmunity, and studies have found that $\mathrm{SCH}$ is associated with dyslipidemia, insulin resistance and renal dysfunction [36]. It was found in this study that the positive rate of thyroid antibody in $\mathrm{SCH}$ group was significantly higher than that in EUTH group. Although there was no significant difference, $\mathrm{SCH}$ group had higher FBG, HbA1c, TG, TC, LDL-C, UA and lower HDL-C than EUTH group. In order to clarify whether the relationship between lifestyle and $\mathrm{SCH}$ was affected by these factors, we analyzed the lifestyle factors that may affect $\mathrm{SCH}$ by logistic regression.

There were some limitations in the present study. First, the study was a cross-sectional study, which can not clarify the causal relationship between lifestyle and $\mathrm{SCH}$. Second, the subjected included in this study was small and all Han ethnicity from Fujian province, China with overall adequate iodine nutrition, which may be accompanied by selection bias. Therefore, large, prospective multicenter studies in different regions, nations and races should be conducted, and the different iodine nutrition statues should be considered.

\section{Conclusions}

In this study, a community-based cross-sectional study was conducted to analyze the relationship between lifestyle and thyroid function in $\mathrm{SCH}$. It was found that lifestyle including sleep, smoking, diet and exercise was closely related to $\mathrm{SCH}$. Staying up late on weekends, exercise, iodine excess and smoking were the influencing factors of thyroid function in $\mathrm{SCH}$.

\section{Abbreviations}

SCH: Subclinical hypothyroidism; TSH: Thyroid stimulating hormone; FT4: Free thyroxine; HPT: Hypothalamus- Pituitary- Thyroid; TPOAb: Thyroid Peroxidase Antibody; TgAb: Thyroglobulin Antibody; BMI: Body mass index; WC: Waist circumference; WHtR: Waist height ratio; SPINA-GT: Thyroid' s secretory capacity; TSHI: Jostel' s TSH index; TTSI: Thyrotroph thyroid hormone resistance index; SBP: Systolic blood pressure; DBP: Diastolic blood pressure; FBG: Fasting blood glucose; HbA1c: Glycosylation hemoglobin; TG: Triglyceride; TC: Total cholesterol; LDL-C: Low density lipoprotein cholesterol; HDL-C: High density lipoprotein cholesterol; PSQI: Pittsburg sleep quality index scale; UIC: Urinary iodine

\section{Supplementary Information}

The online version contains supplementary material available at https://doi. org/10.1186/s12902-021-00772-z

\section{Additional file 1.}

\section{Acknowledgements}

We would like to thank all volunteers who participated in the present study, and all healthcare professionals and staff at the coordinating community. We would also like to express our gratitude to all the members of the research group of the national survey of thyroid diseases and iodine nutrition status (Tide) and Pro. Weiping Teng and Pro. Zhongyan Shan (The First Hospital of China Medical University) who provided support in this study. 


\section{Authors' contributions}

K.W., Y.Z. and L.L. designed the study protocol, performed the data analysis. S.K., J.H., X.G., B.L., X.L. (Xiaoying Lin), L.W. (Lijuan WU), X.L. (Xiaohong Liu), X.L. (Xiaoying Liu) and L.M collected the data. Y.Z., K.W., J.X. and C.X. drafted the manuscript. L.W. (Linxi Wang), L.W. (Li Wu) and Y.W. offered intellectual input and provided critical revision of the manuscript. All authors read and approved the final version of the manuscript.

\section{Funding}

This work was supported by the Special Financial Funds of Fujian Province (Project: Epidemiological Investigation of Thyroid Disease in Fujian, Grant number 2016B019), the Fujian Science and Technology Innovation Joint Fund Project (Project: Study for multiple risk factors intervention and metabolic model of type 2 diabetes mellitus in Fujian Province; Grant number 2017Y9060) and the Research Fund for Public Welfare from National Health and Family Planning Commission of China (Grant number 201402005).

\section{Availability of data and materials}

The data used to support the findings of this study are available from the corresponding author upon reasonable request.

\section{Declarations}

\section{Ethics approval and consent to participate}

All subjects gave written informed consent. The protocol was approved by the Ethics Committee of Fujian Medical University Union Hospital (Grant no.2015KY032).

\section{Consent for publication}

Not applicable.

\section{Competing interests}

Authors have no conflict of interests.

\section{Author details}

'Department of Endocrinology and Metabolism, Fujian Medical University Union Hospital, 29 Xinquan Road, Fujian 350001 Fuzhou, China. ${ }^{2}$ Department of Clinical Pharmacy and Pharmacy Administration, School of Pharmacy, Fujian Medical University, 350122 Fuzhou, Fujian, China.

Received: 18 December 2020 Accepted: 17 May 2021

Published online: 28 May 2021

\section{References}

1. Taylor PN, Albrecht D, Scholz A, Gutierrez-Buey G, Lazarus JH, Dayan CM, Okosieme OE: Global epidemiology of hyperthyroidism and hypothyroidism. Nat Rev Endocrinol 2018, 14(5):301-316.

2. Cheserek MJ, Wu G, Shen L, Shi Y, Le G: Evaluation of the relationship between subclinical hypothyroidism and metabolic syndrome components among workers. Int J Occup Med Environ Health 2014, 27(2):175-187.

3. Delitala AP, Scuteri A, Maioli M, Mangatia P, Vilardi L, Erre GL: Subclinical hypothyroidism and cardiovascular risk factors. Minerva Med 2019, 110(6): 530-545.

4. Teng X, Shan Z, Chen Y, Lai Y, Yu J, Shan L, Bai X, Li Y, Li N, Li Z et al: More than adequate iodine intake may increase subclinical hypothyroidism and autoimmune thyroiditis: a cross-sectional study based on two Chinese communities with different iodine intake levels. Eur J Endocrinol 2011, 164(6):943-950

5. Kim W, Lee J, Ha J, Jo K, Lim D-J, Lee J-M, Chang S-A, Kang M-I, Kim M-H: Association between Sleep Duration and Subclinical Thyroid Dysfunction Based on Nationally Representative Data. J Clin Med 2019.

6. Ravaglia G, Forti P, Maioli F, Pratelli L, Vettori C, Bastagli L, Mariani E, Facchini A, Cucinotta D: Regular moderate intensity physical activity and blood concentrations of endogenous anabolic hormones and thyroid hormones in aging men. Mech Ageing Dev 2001, 122(2):191-203.

7. Tanriverdi A, Ozcan Kahraman B, Ozsoy I, Bayraktar F, Ozgen Saydam B, Acar S, Ozpelit E, Akdeniz B, Savci S: Physical activity in women with subclinical hypothyroidism. J Endocrinol Invest 2019, 42(7):779-785.
8. Goede SL, Leow MK-S, Smit JWA, Dietrich JW: A novel minimal mathematical model of the hypothalamus-pituitary-thyroid axis validated for individualized clinical applications. Math Biosci 2014, 249:1-7.

9. Dietrich JW, Landgrafe-Mende G, Wiora E, Chatzitomaris A, Klein HH, Midgley JEM, Hoermann R: Calculated Parameters of Thyroid Homeostasis: Emerging Tools for Differential Diagnosis and Clinical Research. Front Endocrinol (Lausanne) 2016, 7:57-57.

10. Hoermann R, Midgley JEM, Larisch R, Dietrich JW: The role of functional thyroid capacity in pituitary thyroid feedback regulation. Eur J Clin Invest 2018, 48(10):e13003.

11. Li Y, Teng D, Ba J, Chen B, Du J, He L, Lai X, Teng X, Shi X, Li Y et al: Efficacy and Safety of Long-Term Universal Salt Iodization on Thyroid Disorders: Epidemiological Evidence from 31 Provinces of Mainland China. Thyroid 2020, 30(4):568-579

12. Fadeyev W: Clinical Practice Guidelines for Hypothyroidism in Adults: Co-sponsored by the American Associationof Clinical Endocrinologists and the American Thyroid Association. Clinical and experimental thyroidology 2012, 8(3).

13. WHO, ICCIDD: Assessment of lodine Deficiency Disorders and Monitoring Their Elimination: A Guide for Programme Managers 3rd ed. World Health Organization Geneva 2007, Switzerland.

14. Dietrich JW, Fischer M, Jauch J, Pantke E, Gaertner R, Pickardt C: SPINATHYR: A novel systems theoretic approach to determine the secretion capacity of the thyroid gland. European Journal of Internal Medicine 1999, 10 (Suppl. 1):S34.

15. Jostel A, Ryder WD, Shalet SM: The use of thyroid function tests in the diagnosis of hypopituitarism: definition and evaluation of the TSH Index. Clin Endocrinol (Oxf) 2009, 71(4):529-534.

16. Chatzitomaris A, Köditz R, Höppner W, Peters S, Klein HH, Dietrich JW: A novel de novo mutation in the thyroid hormone receptor-beta gene. Experimental and Clinical Endocrinology \& Diabetes 2015, 122(03).

17. Pohlenz J, Weiss RE, Macchia PE, Pannain S, Lau IT, Ho H, Refetoff S: Five new families with resistance to thyroid hormone not caused by mutations in the thyroid hormone receptor beta gene. J Clin Endocrinol Metab 1999, 84(11):3919-3928.

18. Buysse DJ, Reynolds CF, 3rd, Monk TH, Berman SR, Kupfer DJ: The Pittsburgh Sleep Quality Index: a new instrument for psychiatric practice and research. Psychiatry Res 1989, 28(2):193-213.

19. Kuhs H, Farber D, Tolle R: Serum prolactin, growth hormone, total corticoids, thyroid hormones and thyrotropine during serial therapeutic sleep deprivation. Biol Psychiatry 1996, 39(10):857-864

20. Russell W, Harrison RF, Smith N, Darzy K, Shalet S, Weetman AP, Ross $\mathrm{RJ}$ : Free triiodothyronine has a distinct circadian rhythm that is delayed but parallels thyrotropin levels. J Clin Endocrinol Metab 2008, 93(6): 2300-2306.

21. Ikegami K, Refetoff S, Van Cauter E, Yoshimura T: Interconnection between circadian clocks and thyroid function. Nat Rev Endocrinol 2019, 15(10):590-600

22. Parker DC, Rossman LG, Pekary AE, Hershman JM: Effect of 64-hour sleep deprivation on the circadian waveform of thyrotropin (TSH): further evidence of sleep-related inhibition of TSH release. J Clin Endocrinol Metab 1987, 64(1):157-161.

23. Ikegami $K$, Liao XH, Hoshino $Y$, Ono H, Ota W, Ito $Y$, Nishiwaki-Ohkawa $T$, Sato C, Kitajima K, ligo M et al: Tissue-specific posttranslational modification allows functional targeting of thyrotropin. Cell Rep 2014, 9(3):801-810.

24. Kadoya M, Koyama S, Morimoto A, Miyoshi A, Kakutani M, Hamamoto K, Kurajoh M, Shoji T, Moriwaki Y, Koshiba M et al: Serum Macro TSH Level is Associated with Sleep Quality in Patients with Cardiovascular Risks - HSCAA Study. Sci Rep 2017, 7:44387.

25. Werneck FZ, Coelho EF, Almas SP, Garcia M, Bonfante HLM, Lima JRP, Vigario PDS, Mainenti MRM, Teixeira P, Vaisman M: Exercise training improves quality of life in women with subclinical hypothyroidism: a randomized clinical trial. Arch Endocrinol Metab 2018, 62(5):530-536.

26. Ahn N, Kim HS, Kim K: Exercise training-induced changes in metabolic syndrome parameters, carotid wall thickness, and thyroid function in middle-aged women with subclinical hypothyroidism. Pflugers Arch 2019, 471(3):479-489.

27. Booms S, Hill E, Kulhanek L, Vredeveld J, Gregg B: Iodine Deficiency and Hypothyroidism From Voluntary Diet Restrictions in the US: Case Reports. Pediatrics 2016, 137(6)

28. Nepal AK, Suwal R, Gautam S, Shah GS, Baral N, Andersson M, Zimmermann MB: Subclinical Hypothyroidism and Elevated Thyroglobulin in Infants with Chronic Excess lodine Intake. Thyroid 2015, 25(7):851-859. 
29. Suzuki K, Kimura H, Wu H, Kudo N, Kim WB, Suzuki S, Yoshida A, Caturegli P, Kohn LD: Excess iodide decreases transcription of NIS and VEGF genes in rat FRTL-5 thyroid cells. Biochem Biophys Res Commun 2010, 393(2):286-290.

30. Dietrich JW, Tesche A, Pickardt CR, Mitzdorf U: Thyrotropic Feedback Control: Evidence for an Additional Ultrashort Feedback Loop from Fractal Analysis. Cybernetics and Systems 2010, 35(4):315-331.

31. Markou K, Georgopoulos N, Kyriazopoulou V, Vagenakis AG: lodine-Induced hypothyroidism. Thyroid 2001, 11(5):501-510.

32. Katagiri R, Asakura K, Sasaki S, Hirota N, Notsu A, Miura A, Todoriki H, Fukui M, Date C: Estimation of habitual iodine intake in Japanese adults using 16 $\mathrm{d}$ diet records over four seasons with a newly developed food composition database for iodine. Br J Nutr 2015, 114(4):624-634

33. Muller B, Zulewski H, Huber P, Ratcliffe JG, Staub JJ: Impaired action of thyroid hormone associated with smoking in women with hypothyroidism. N Engl J Med 1995, 333(15):964-969.

34. Knudsen N, Bülow I, Laurberg P, Perrild H, Ovesen L, Jørgensen T: High occurrence of thyroid multinodularity and low occurrence of subclinical hypothyroidism among tobacco smokers in a large population study. J Endocrinol 2002, 175(3):571-576.

35. Carle A, Bulow Pedersen I, Knudsen N, Perrild H, Ovesen L, Banke Rasmussen L, Jorgensen T, Laurberg P: Smoking cessation is followed by a sharp but transient rise in the incidence of overt autoimmune hypothyroidism - a population-based, case-control study. Clin Endocrinol (Oxf) 2012, 77(5):764-772

36. Biondi B, Cappola AR, Cooper DS: Subclinical Hypothyroidism: A Review. JAMA 2019, 322(2):153-160.

\section{Publisher's Note}

Springer Nature remains neutral with regard to jurisdictional claims in published maps and institutional affiliations.

Ready to submit your research? Choose BMC and benefit from:

- fast, convenient online submission

- thorough peer review by experienced researchers in your field

- rapid publication on acceptance

- support for research data, including large and complex data types

- gold Open Access which fosters wider collaboration and increased citations

- maximum visibility for your research: over $100 \mathrm{M}$ website views per year

At BMC, research is always in progress.

Learn more biomedcentral.com/submissions 